\title{
DETERMINACIÓN DE FACTORES DE CORRECCIÓN ALTITUDINAL PARA MODELOS DIGITALES DE ELEVACIÓN
}

\section{DETERMINATION OF ALTITUDE CORRECTION FACTORS FOR DIGITAL ELEVATION MODEL}

\author{
Sr. Rodrigo Esteban González G. ${ }^{1}$
}

\section{RESUMEN}

La utilización de los Modelos Digitales de Elevación (MDE) imparte una necesidad en comprobar su exactitud y precisión, debido a la utilización en una variedad de aplicaciones, ya sea en proyectos privados y públicos. Para tal efecto, se revela la necesidad de comprender el aporte que entrega la geodesia como una ciencia básica, orientada en los resultados de las mediciones y cálculos de las dimensiones que tiene la tierra. En efecto, en este estudio se pretende determinar la exactitud vertical, a partir de la asignación y comparación altimétrica entre 4 nivelaciones geométricas con 3 MDE de fuente abierta: SRTM, ASTER GDEM y PALSAR. Asimismo, se analizaron, los modelos geopotenciales de estándar mundial (EGM-96 y EGM-2008), en post de comprobar a, cuál sistema de referencia vertical pertenece cada modelo. Por lo tanto, el objetivo planteado en este artículo refiere a la determinación de factores de corrección altitudinal local, mediante la utilización de la ecuación de observación, esta, permite determinar valores más probables, basados en el principio de Mínimos Cuadrados, en este estudio se implementó dicha ecuación, para finalmente obtener factores para corregir la exactitud vertical altimétrica. En relación con el análisis, este, se llevó a cabo sobre la base de un conjunto de 132 muestras, las cuales se encuentra localizadas, próximo a una trayectoria lineal de alrededor de $116 \mathrm{~km}$.

Palabras Claves: Modelo Digital de elevación, Factores de Corrección, Comparación altimétrica.

\section{ABSTRACT}

Using Digital Elevation Models (DEMs) imparts a necessity for checking its accuracy and precision, due to their variety of applications, whether in private or public projects. For this purpose, understanding the contribution of geodesy as a basic science, oriented towards the results earth measurements and calculations, is revealed. Therefore, vertical accuracy this study aims determinate from assignment and altimetric comparison between 4 geometric levelling considering 3 open source DEMs: SRTM, ASTER GDEM and PALSAR. Also, worlds standard geopotential models (EGM-96 and EGM-2008) were analyzed to verify specific vertical reference system adjustment for each model. Then, the objective proposed in the present work describes a methodology for determination of local altitudinal correction factors, conditioned by the use of an observation equation, which allows determining more probable values, based on the principle of Least Squares, this means, the equation was implemented, and factors were obtained to correct the vertical altimeter accuracy.

Regarding the analysis, this was carried out based on a set of 132 samples, which are located close to a 116 $\mathrm{km}$ linear trajectory.

KeyWords: Digital Elevation Model, Correction Factors, Altimetric Comparison.

\footnotetext{
${ }^{1}$ Ingeniería en Geomensura y Cartografía, Universidad Bernardo O’Higgins, rgonzalez@srk.cl
} 


\section{INTRODUCCIÓN}

En esta investigación se aborda la precisión y calidad de modelos digitales de elevación $\left(\mathrm{MDE}^{2}\right)$ de licencia abierta. Estos MDE proporcionan datos esenciales en topografía para analizar las alturas, depresiones, cuencas y llanuras de la superficie a escalas de la tierra. El acceso a los MDE a nivel global se ha incrementado a partir de la década de los 90, entre otras razones, gracias al mayor desarrollo tecnológico y al aumento de las misiones topográficas tales como: SRTM ${ }^{3}$, ASTER GDEM $^{4}$ y ALOS PALSAR ${ }^{5}$, desarrolladas por Agencias Espaciales globales (Menegbo E. M, 2015); (Alganci et al., 2018).

La presencia de satélites de observación terrestre juega un rol muy relevante en el desarrollo de los trabajos de investigación científica, en el ámbito privado o público, ya que proporcionan, de manera inteligente y continua, datos de valioso contenido. Sus sensores presentan características esenciales que permiten dar una cobertura global para obtener un análisis desde el punto de vista local y regional. Entre toda la diversidad de datos suministrados por satélites de observación, los de elevación terrestre son los que alcanzan especial relevancia para este estudio, porque con ellos se generan MDE (Rodríguez \& Barrena, 2006).

"Estos modelos corresponden a una estructura numérica de datos los cuales representan la distribución espacial de la altitud de la superficie del terreno y permite identificar unidades de relieve con características comunes y significado geomorfológico." (Felicísimo, 1994).

El desarrollo de las misiones y su aplicación ha contribuido particularmente en aspectos como una mayor accesibilidad a los datos y menor costo en la elaboración digital a través de procesamiento con softwares tales como: ArcGis, Qgis, Erdas Imagine, Envi, GlobalMapper,Autocad Civil, entre otros.

2 Modelo Digital de Elevación

3 Shuttle Radar Topography Mission

4 ASTER Global Digital Elevation Map
Como complemento, esto lleva a verificar la caracterización de los elementos que afectan la variación altitudinal en un MDE, ya que es un componente fundamental para determinar los factores que intervienen en la precisión y el cual, define los alcances en la utilización de dicha información tridimensional propia en trabajos de investigación.

Atendiendo a estas consideraciones, en el desarrollo de este estudio, se realizaron búsquedas de publicaciones científicas, con el objetivo de fortalecer el tema abordado, como resultado, se puede indicar que actualmente en el contexto de Chile existe escasa publicación de investigaciones científicas con respecto a la evaluación de precisión y calidad de los MDE para algunas zonas específicas de Chile. Como complemento, sobre la validez, hay que indicar que en el ámbito militar y defensa la precisión de estos modelos es vital en artillería y empleo en asuntos bélicos.

En el siglo XXI, la utilización de la geotecnología con precisión es fundamental para el entendimiento de los investigadores que desarrollan estudios en relación con el conocimiento de las ciencias de la Tierra. La geodesia constituye una ciencia básica para otras disciplinas, como la topografía, fotogrametría, cartografía, ingeniería civil, navegación, SIG (sistemas de información geográfica), sin olvidar otros tipos de fines como los militares. (IGN, 2014). A causa de ello, se comprende que la geodesia responderá inquietudes sobre la forma de la tierra y además apoyará en identificar los diferentes factores que influyen en la precisión y calidad para cada MDE. Porque es una ciencia básica que usa los principios de las matemáticas, la astronomía y la física que actualmente se aplica con el uso de modernas tecnologías. (Bolla, 2009)

A partir de la observación de los MDE con modelos de gravedad global o geopotencial y de la

5 Advanced Land Observation Satellite - Phased Array type Lband Synthetic Aperture Radar

Revista Geográfica de Chile Terra Australis, Vol.56 (2020) 110-128, ISSN: 0378-8482 - EISSN: 0719-9562 
utilización de líneas de nivelación geométrica, es altamente viable y factible obtener un error o factor de desplazamiento. Por lo tanto, esta publicación se plantea como objetivo determinar factores de corrección altitudinal local, evaluando su precisión y calidad altimétrica, utilizando una comparación de los MDE disponibles (SRTM, ASTER GDEM y ALOS PALSAR), contrastándolo con el levantamiento altimétrico (nivelación geométrica de precisión) y los modelos geopotenciales.

Para tal efecto, se alcanzarán indicadores de diferencias a partir de dicho análisis. Al mismo tiempo se corrobora el datum vertical que corresponde cada uno, obteniendo también, parámetros locales a partir de análisis estadísticos que optimice la corrección en función de la calibración de cada modelo.

\section{MATERIALES Y MÉTODOS}

\section{ÁREA DE ESTUDIO}

El trabajo está elaborado en la zona central del territorio de Chile, y fue determinado por cuatro líneas de nivelación geométricas, cedidas por parte del Departamento de Geodesia del IGM . Estas 4 nivelaciones comprenden parte del territorio estudiado, la primera se emplaza desde la Región de Valparaíso, incluyendo su trayectoria en la comuna de Pudahuel, Estación Central y Santiago, dentro de la Región Metropolitana de Santiago este tramo se denomina "ValparaísoSantiago". La Segunda inicia en la comuna de Colina y continua en Rinconada y termina en la comuna de Los Andes, este tramo se denomina "Santiago-Los Andes". La Tercera inicia en la comuna de Los Andes y continua en San Esteban y termina en un sector denominado Río Blanco, este tramo se denomina "Los Andes-Río Blanco". El cuarto y última nivelación, inicia en el Sector de Río Blanco en la comuna de Los Andes y continua en el sector de Portillo y termina en un sector denominado Túnel Cristo redentor, este tramo se denomina "Río Blanco-Portillo", la denominación de las nivelaciones geométricas difiere de la

\footnotetext{
${ }^{6}$ Instituto Geográfico Militar, Servicio oficial, técnico y
permanente del Estado en todo lo referido a la cartografía y ${ }^{6}$ Instituto Geográfico Militar, Servicio oficial, técnico y
permanente del Estado en todo lo referido a la cartografía y levantamiento del territorio nacional.(Chile)
}

Revista Geográfica de Chile Terra Australis, Vol.56 (2020) 110-128, ISSN: 0378-8482 - EISSN: 0719-9562 valores de altura referidos a una superficie de diferentes técnicas o métodos".(Mancero et al., 2015). Por otra parte, se resalta la importancia de la distribución espacial de la variable representada (Felicísimo, 1994) Por contraste, existe según lo que representa $\mathrm{DSM}^{7}$, este, es Similar a un MDE o MDT, pero muestra la parte superior de todas las superficies incluyendo edificios, árboles y otras características por encima de la tierra desnuda. (Felicísimo, 1994)

\section{SISTEMA DE ALTURA}

Según SIRGAS, (2019).En el contexto de América Latina, los datum verticales utilizados actualmente se refieren a diferentes mareógrafos, de acuerdo con Sánchez, (2007) indica que el posicionamiento de los mareógrafos esta determinados por el Sistema GNSS, para identificar movimientos verticales de la corteza y cambios de nivel del mar. La superficie de referencia altimétrica es el geoide, el cual está definido como la superficie equipotencial (de igual valor de atracción gravitacional) que coincide con la superficie de los océanos en reposo, extendida sobre los continentes, su denominación más común es Nivel Medio del Mar - NMM. La altura sobre el geoide (o sobre el NMM) se denomina "altura ortométrica", también referida como altitud 0 elevación.(Zepeda,2014)

\footnotetext{
${ }^{7}$ Digital Surface Model, Modelo Digital de Superficie
} referencia o datum vertical obtenidos mediante 


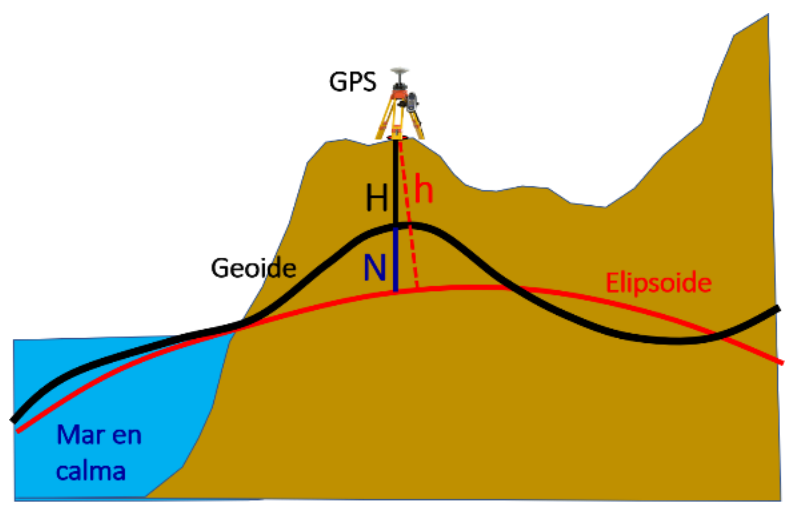

Figura 1 Esquema de Alturas

De acuerdo con la Figura 1, se consideran diferentes tipos de alturas como la Altura o Ondulación Geoidal (N) que es aquella que representa en un punto la altura del geoide respecto al elipsoide. Por lo tanto, se hace necesario el conocimiento de este valor para la reducción de alturas elipsoidales a alturas NMM, según (Zepeda, 2014) se deduce que (Ecuación 1)

$$
h=H+N(1)
$$

La altura elipsoidal (h) es la altura calculada desde el punto en la superficie terrestre al elipsoide que pasa por la normal al elipsoide en dicho punto (en el caso tridimensional). En geodesia se usa un elipsoide que óptimamente se aproxima al geoide según la definición Gauss-Listing, o sea que coincide con el nivel medio del mar (en calma). (SNIT, 2018).

La altura ortométrica, que se define como "valor promedio de la gravedad real medida a lo largo de la línea de la plomada entre el geoide y el punto de observación". (Sánchez, SIRGAS, 2002). Esto se resuelve con el despeje de la (Ecuación 2):

$$
\mathrm{H}=\mathrm{h}-\mathrm{N}(2)
$$

Donde:

$\mathrm{H}=$ Altura ortométrica $\mathrm{h}=$ Altura elipsoidal $\mathrm{N}=$ Ondulación Geoidal. 


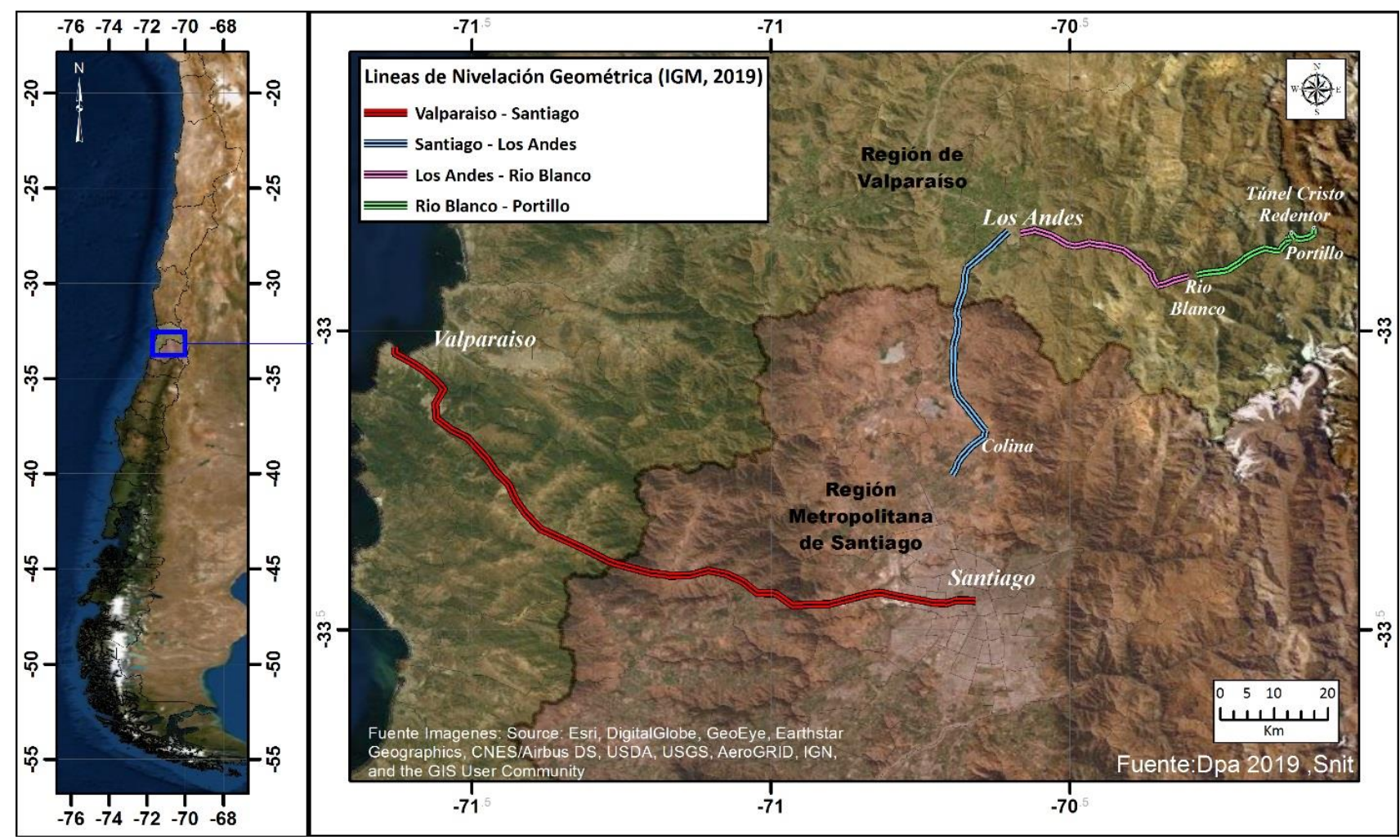

Figura 2 Localización General Fuente: Elaboración Propia

\section{DATOS UTILIZADOS}

\section{NIVELACIÓN GEOMÉTRICA}

La nivelación geométrica es el método más preciso para la obtención de desniveles. (Zepeda, 2014)(ver Figura 3)

Esta permite determinar elevaciones con resultados de mayor calidad en el proceso de monitoreo. Dicha técnica in situ, usa equipos de alta precisión (niveles de precisión, miras industriales o de INVAR ${ }^{8}$ ) (Francoso et al., 2019).

Figura 3 Esquema de desniveles (Zepeda, 2014)

En Chile, el IGM es el servicio oficial que regulariza, la Red de Nivelación nacional, la cual, está compuesta por alrededor de 5.000 puntos,

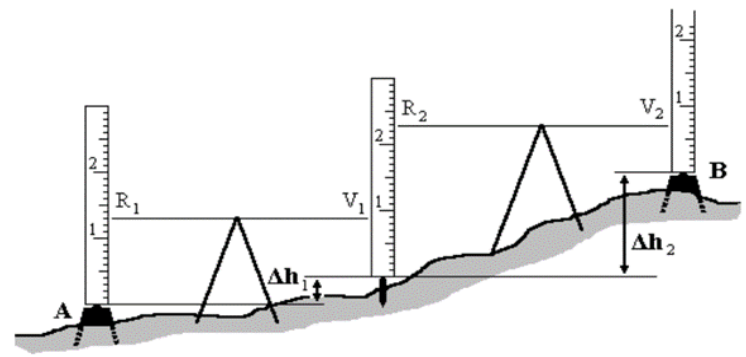

situados en la trayectoria de las carreteras del país. Dichas nivelaciones se encuentran monumentadas y referidas a una altura Nivel Medio del Mar. La altura NMM, pertenece al sistema de referencia vertical oficial del país. Para esta investigación, es importante indicar que el Departamento de Geodesia del (IGM, 2019)

\footnotetext{
${ }^{8}$ Miras de Nivelación, resulta idóneo como soporte de escalas, pues su longitud no varía con los cambios de temperatura
}

Revista Geográfica de Chile Terra Australis, Vol.56 (2020) 110-128, ISSN: 0378-8482 - EISSN: 0719-9562 
facilitó 4 líneas de nivelación para uso académico, las cuales comprendían: 1) Valparaíso-Santiago 2) Santiago-Los Andes 3) Los Andes-Río Blanco y 4) Río Blanco Portillo. (Figura 2).El Nivel medio del Mar, es determinado por una red de Mareógrafos distribuidos a lo largo de las costas de Chile, administrados por el $\mathrm{SHOA}^{9}$, quién es el organismo responsable en determinar la "cota fija de marea" que el IGM toma como inicio para la densificación de sus redes de nivelación hacia el continente. (Saez, 2019).

\section{MODELOS DIGITALES DE ELEVACIÓN SRTM (SHUTTLE RADAR TOPOGRAPHY MISSION)}

Proyecto conjunto entre NIMA $^{10}$ y la NASA ${ }^{11}$ y las agencias espaciales de Alemania (DLR) e Italia (ASI). La misión tuvo como objetivo generar datos topográficos para el $100 \%$ de la superficie de la tierra. Con puntos ubicados en una grilla de 1 arco-segundo (30 m aproximadamente) en latitud y longitud (Hirt, Filmer, \& Featherstone, 2010). Utilizó un sistema en banda $C$ en modo ScanSAR cubriendo un ancho de barrido de $225 \mathrm{~km}$, otra banda $X$ con un ancho de barrido de $50 \mathrm{~km}$ utilizando interferometría ${ }^{12}$ (Hoffmann, J. and D. Walter, 2006).

\section{ASTER GDEM (RADIÓMETRO ESPACIAL DE EMISIÓN Y REFLEXIÓN TÉRMICA AVANZADO)}

Satélite que comprende un sensor óptico de imágenes elaborado y ejecutado por el Ministerio de Economía, Comercio e industria (METI) de Japón, e ubicado en el satélite de la NASA (TERRA). Algunas Características principales: Cobertura mundial, con una resolución espacial nominal de $30 \mathrm{~m}$. El MDE se crea en función de la estereoscopía de las bandas 3N (Vista a Nadir) y 3B (Vista retrasada) alcanzando modelos 1)digital relativo que se desarrolla sin puntos de control y otro 2)digital absoluto que utiliza puntos de

\footnotetext{
${ }^{9}$ Servicio Hidrográfico y Oceanográfico de la Armada de Chile.

${ }^{10}$ National Imagery and Mapping Agency desde 1996 a 2003 actualmente (NGA) National Geospatial-Intelligence Agency. (Estados Unidos de Norteamérica)
}

control. (Burgos, 2012) Los errores medios cuadráticos en la vertical fluctúan entre los 10 y 25 m (ERSDAC, 2003).

\section{ALOS (ADVANCED LAND OBSERVING SATELLITE)}

El programa satelital japonés de observación de la tierra consiste en dos series de satélites conformados de acuerdo con el objetivo de observación. La primera serie de satélites está principalmente diseñada para observaciones atmosféricas y marinas, mientras que la segunda serie para observaciones del suelo. El satélite "Advanced Land Observing Satellite ALOS" es el sucesor del satélite "Japanese Earth Resources Satellite-1 JERS-1" (banda L, polarización HH, $35^{\circ}$ ángulo off-nadir) en operación durante 1992 1998. ALOS fue exitosamente lanzado desde el Centro Espacial de Tanegashima-Japón en enero/24/2006 (Rosenqvist, Shimada, \& Watanabe, 2004), (Jaxa, 2008). En mayo del 2011, JAXA anunció la terminación de la vida útil del satélite ALOS y se anuncia que para el 2013 el lanzamiento de ALOS-2 con el mejoramiento de sus modos de adquisición, mejor resolución, más ángulos de incidencia, etc.

ALOS utiliza técnicas avanzadas de observación del suelo con los siguientes objetivos:

1.Proveer mapas para Japón y países contenidos en la región Asia-Pacífico.

2.Realizar observaciones regionales para fomentar el equilibrio entre el planeta y el desarrollo.

3.Monitorear desastres alrededor del mundo.

4. Investigar recursos naturales.

5.Desarrollar tecnología para futuros satélites de observación de la Tierra. (Torres Mayorga, 2013).

\footnotetext{
${ }^{11}$ Administración Nacional de Aeronáutica y del Espacio de Estados Unidos de Norteamérica.

${ }^{12}$ Técnica en radar que consiste en lograr la altura de un punto sobre el terreno, a partir de dos imágenes SAR obtenidas desde puntos próximos.
} 


\section{MODELOS GEOPOTENCIALES}

Con el avance en la tecnología GNSS $^{13}$ existe la necesidad de desarrollar modelos del geoide regionales o globales altamente precisos. Por lo tanto, si se dispone de modelos que nos provean de valores en la ondulación del geoide con la misma precisión, se alcanzará a calcular altitudes ortométricas precisas que es lo que se demanda en la mayor parte de los trabajos realizados por las instituciones públicas y privadas. (Martinez Toro \& Bethencourt Fernandez, 2012)

En la tabla 1 se resumen características generales sobre las misiones topográficas.

\begin{tabular}{|c|c|c|c|}
\hline & SRTM V3 & $\begin{array}{c}\text { ASTER } \\
\text { (GDEM V3) }\end{array}$ & PALSAR \\
\hline $\begin{array}{l}\text { Agencia } \\
\text { Espacial }\end{array}$ & NASA & $\begin{array}{l}\text { NASA y } \\
\text { METI }\end{array}$ & $\begin{array}{l}\text { NASA y } \\
\text { JAXA }\end{array}$ \\
\hline $\begin{array}{c}\text { Periodo } \\
\text { adquisición de } \\
\text { información }\end{array}$ & 2000 & desde 1999 & 2006 a 2011 \\
\hline Cobertura & $56^{\circ} \mathrm{S}$ a $60^{\circ} \mathrm{N}$ & $83^{\circ} \mathrm{S}$ a $83^{\circ} \mathrm{N}$ & \\
\hline $\begin{array}{l}\text { Ancho de } \\
\text { Barrido }\end{array}$ & $\begin{array}{l}\text { Banda C } \\
(225 \mathrm{~km}) / \\
\text { Banda X } \\
(50 \mathrm{~km})\end{array}$ & $60 \mathrm{~km}$ & \\
\hline $\begin{array}{l}\text { Tamaño de } \\
\text { Píxel }\end{array}$ & $\begin{array}{c}1 \text { arco } \\
\text { segundo } \\
\text { (aprox. 30m) }\end{array}$ & $\begin{array}{c}1 \text { arco } \\
\text { segundo } \\
\text { (aprox. } 30 \mathrm{~m} \text { ) }\end{array}$ & $12.5 \mathrm{~m}$ \\
\hline Sensor & Radar & Óptico & Radar \\
\hline $\begin{array}{c}\text { Método de } \\
\text { Procesamiento }\end{array}$ & $\begin{array}{l}\text { Interferomet } \\
\text { ría SAR }\end{array}$ & $\begin{array}{c}\text { Imágenes } \\
\text { Estereoscóp } \\
\text { icas }\end{array}$ & $\begin{array}{c}\text { Interferometr } \\
\text { ía SAR }\end{array}$ \\
\hline $\begin{array}{c}\text { Datum } \\
\text { Horizontal }\end{array}$ & Wgs84 & Wgs84 & Wgs84 \\
\hline Datum Vertical & Egm-96 & Egm-96 & Egm-2008 \\
\hline $\begin{array}{c}\text { Sistema } \\
\text { Coordenado }\end{array}$ & $\begin{array}{c}\text { Coordenada } \\
\text { s } \\
\text { Geográficas }\end{array}$ & $\begin{array}{l}\text { Coordenada } \\
\text { s } \\
\text { Geográficas }\end{array}$ & $\begin{array}{c}\text { Coordenada } \\
\text { s } \\
\text { Geográficas }\end{array}$ \\
\hline Formato DEM & $\begin{array}{c}\text { Geotiff,dem, } \\
\text { Ascii }\end{array}$ & $\begin{array}{l}\text { Geotiff dem, } \\
\text { Ascii }\end{array}$ & $\begin{array}{c}\text { Geotiff dem, } \\
\text { Ascii }\end{array}$ \\
\hline $\begin{array}{l}\text { P. }\left({ }^{\star}\right) \text { Vertical } \\
\text { relativa }\end{array}$ & $10 \mathrm{~m}$ & $10 \mathrm{~m}$ & $9 \mathrm{~m}$ \\
\hline $\begin{array}{l}\text { P. }\left({ }^{\star}\right) \text { Vertical } \\
\text { absoluta }\end{array}$ & $16 \mathrm{~m}$ & $7 \mathrm{~m}$ & $5 \mathrm{~m}$ \\
\hline $\begin{array}{l}\mathrm{P} .\left(^{\star}\right) \text { Horizontal } \\
\text { relativa }\end{array}$ & $15 \mathrm{~m}$ & $10 \mathrm{~m}$ & $12.5 \mathrm{~m}$ \\
\hline $\begin{array}{l}\text { P. }\left({ }^{*}\right) . \text { Horizontal } \\
\text { absoluta }\end{array}$ & $20 \mathrm{~m}$ & $7 \mathrm{~m}$ & $10 \mathrm{~m}$ \\
\hline
\end{tabular}

${ }^{13}$ Global Navigation Satellite System, sistema global de navegación por satélite.

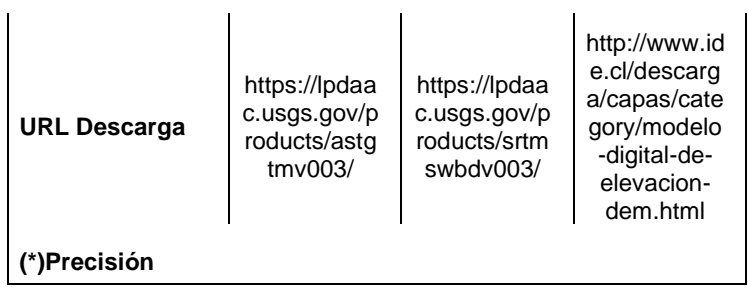

Tabla 1 Descripción Misiones Topográficas Fuente: Elaboración Propia

Al respecto, con los modelos utilizados en este análisis se puede indicar el uso del Modelo EGM96 que consiste en coeficientes armónicos esféricos desarrollados por una colaboración fundamentalmente de la NASA y entre el centro de vuelo espacial Goddard, la $N A^{14}$ y las Universidades de Estados Unidos de los Estados de Ohio y Texas. (Tierra, 2009). En paralelo se utilizó el más actual, que es el EGM-2008 un modelo armónico esférico del potencial gravitatorio de la Tierra desarrollado por la Agencia Nacional de Inteligencia Geoespacial, conformado por información del satélite GRACE y también un conjunto global de anomalías terrestres (terrestres y marinas) de gravedad de aire libre de un área media equivalente a una grilla de 5 minutos de arco (SNIT, 2018).

Los modelos fueron extraídos en formato ZIP de la (NG-IA, 2019), Agencia Nacional de inteligencia geoespacial de Estados Unidos de Norteamérica.

\section{METODOLOGÍA}

La presente investigación se desarrolló con el sistema de referencia en coordenadas geográficas referidas al datum Wgs-84 para el posicionamiento espacial de la información. De acuerdo con la (Figura 4), se aprecia, la metodología empleada y que conforma dicho estudio. De este modo, el análisis operacional, es decir, asignación y comparación espacial, fue

\footnotetext{
${ }^{14}$ National Geospatial Intelligence Agency. (Estados Unidos de Norteamérica)
}

Revista Geográfica de Chile Terra Australis, Vol.56 (2020) 110-128, ISSN: 0378-8482 - EISSN: 0719-9562 
desarrollado en Arcgis $^{15}$ versión 10.7, esta aplicación permite analizar en 2 y 3 dimensiones, contrastando modelos de tipo Vector ${ }^{16}$ con Raster ${ }^{17}$

Para el caso del trabajo se realizaron los siguientes procesos: 1) Extracción de elevación de cada MDE por posición espacial de la

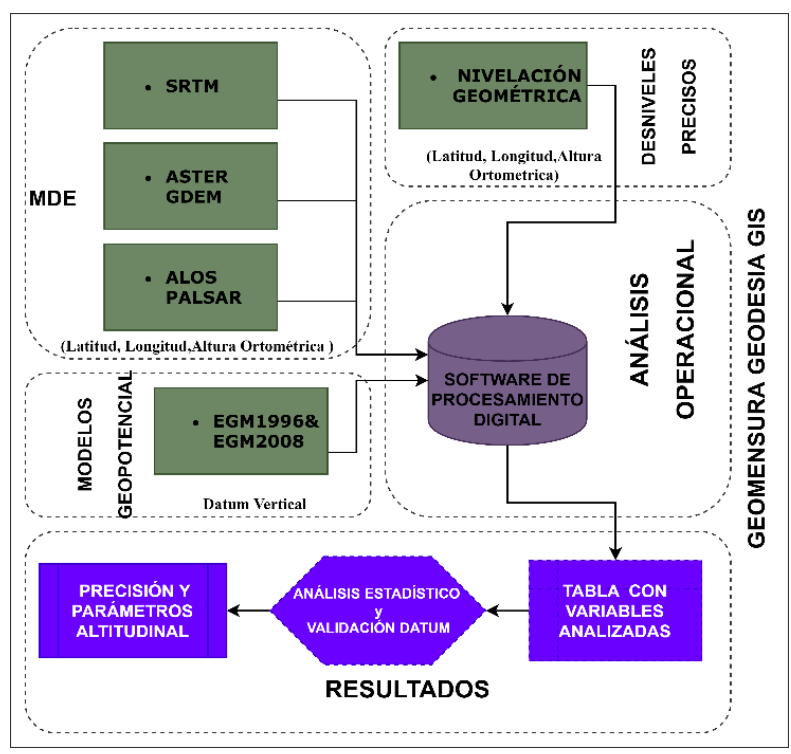

Figura 4 Diagrama de procesos Fuente: Elaboración Propia

Nivelación Geométrica 2) Extracción de valores de los Modelos Geopotenciales por posición espacial de la Nivelación Geométrica. En cada análisis, se obtuvo el valor altimétrico asociado a cada píxel ajustado la medida in situ de la nivelación. Es decir que, dicho proceso accede a evaluar los valores de celda basados en un conjunto de entidades de punto y registra los valores en la tabla de atributos de una clase de

\footnotetext{
${ }^{15}$ Es un completo sistema que permite recopilar, organizar, administrar, analizar, compartir y distribuir información geográfica.

16 Estructura de datos utilizada para almacenar datos geográficos(Puntos, Líneas o Áreas).
}

entidad de salida. (ESRI, 2019).

Posterior al proceso de asignación, cada nivelación, adjunta una tabla de atributos que contiene valores altimétricos, diferenciados por el nombre correspondiente a cada MDE, definido en un campo. Cabe indicar, que además incorpora los datos de los dos modelos geopotenciales. Dichas tablas se exportan como formato .csv para su posterior evaluación en el software Excel. Dicha información se encuentra en el anexo 1. Por consiguiente, se importa y compila la información para llevar a cabo los siguientes procedimientos:

\section{Comparación Altimétrica \\ Estimación de errores \\ Cálculos de Alturas \\ Determinación de factores de ajuste}

Los temas tratados a continuación que incluyen operaciones analíticas fueron aplicados caso a caso. En cuanto a la realización de la comparación altimétrica es decir obtener la diferencia vertical, se evalúa mediante (Véase ecuación 3) (FGDC, 1998)

$$
\boldsymbol{D i f}_{\boldsymbol{z}}=\left(Z_{n n m}-Z_{\text {dem }}\right)
$$

El resultado obtenido determina la precisión vertical relativa. Al mismo tiempo y con el objetivo de generar estadística de precisión vertical, se validan los resultados de comparación altimétrica con el método de estimación de la Raíz del Error Medio Cuadrático $\left(\mathrm{RMSE}^{18}\right)$. Esta comprueba la cuantificación del error existente, entre los conjuntos evaluados. (FGDC, 1998)

$$
\boldsymbol{R M S E}=\sqrt{\frac{\epsilon_{i=1}^{n}\left(Z_{n n m}-Z_{d e m}\right)^{2}}{n}}
$$

Donde:

RMSE: Error Medio Cuadrático

\footnotetext{
${ }^{17}$ En su forma más simple, un ráster consta de una matriz de celdas (o píxeles) organizadas en filas y columnas (o una cuadrícula).

${ }^{18}$ Root MeanSquare Error, Raíz del error medio cuadrático
} 
$\boldsymbol{Z}_{\text {nnm }}$ Elevación del terreno (Nivelación Geométrica)

$\boldsymbol{Z}_{\text {dem }}$ : Elevación Del terreno (MDE analizados)

$n=$ Número de puntos evaluados

A este respecto, se debe considerar que para este estudio se analizó, tanto para SRTM y ASTERGDEM la versión $N^{\circ} 3$ y para PALSAR la versión $\mathrm{N}^{\circ} 1$ la cual fue elaborada por CIREN $^{19}$. En este sentido se comprende que los errores sistemáticos que considera cada MDE, se van eliminando, de acuerdo con técnicas y nuevas modalidades de procesamientos, esto, influye directamente en la creación de nuevas versiones. "Si el error vertical es distribuido normalmente, el factor 1.9600 se aplica para calcular el error lineal al nivel de confianza del 95\%”. (Greenwalt \& Shultz, 1968)

Por lo tanto, la exactitud vertical absoluta (Eva), informada de acuerdo con el NSSDA $^{20}$ se calculará mediante la siguiente fórmula: (FGDC, 1998)

$$
\mathrm{Eva}=1.9600 * \text { RMSE (5) }
$$

Según Garafulic C.,(2011) para determinar factores de ajuste se utilizó un modelo para la interpolación de las ondulaciones geoidales, definen los parámetros de ajustes a partir de la utilización de una función polinomial de mínimo tercer grado que para este caso se utiliza un polinomio de cuarto grado todo esto en función de "y".

Donde (Ecuación 6) (Garafulic C., 2011)

$$
\boldsymbol{L a}=f\left(X_{a}\right)(6)
$$

Donde:

La: Vector de dos valores Observados ajustados $X_{a}$ :Vector dos parámetros ajustados

Modelo liberalizado (Ecuación 7) (Garafulic C., 2011)

$$
\boldsymbol{A} \boldsymbol{X}+\boldsymbol{L}=\boldsymbol{V}(7) \text { ecua }
$$

19 Centro de información de Recursos Naturales (Chile)
Donde:
$\boldsymbol{A X}$ : Vector de corrección
$\boldsymbol{L}:$ Vector de observación
$\boldsymbol{V}:$ Vector de Residuos

Para este artículo se utilizó: (Ecuación 8) (Garafulic C., 2011)

$$
\boldsymbol{f}(\boldsymbol{y})=a+b x+c x^{2}+d x^{3}+e x^{4}(8)
$$

Dicho modelo es el método de ecuación de observación, de acuerdo con Zepeda, 2014, "se tienen más observaciones que incógnitas, lo que permite determinar valores más probables de las incógnitas basados en el principio de Mínimos Cuadrados. Para solución única, el número de ecuaciones es igual al número de incógnitas".

Finalmente, esta ecuación de observación fue realizada en una planilla de Excel, que estará incluida como entrega en un anexo. Este método de regresión polinómica se implementó para determinar los factores locales de ajustes, en efecto, este se aplicó en cada nivelación y también en forma agrupada para obtener mayores resultados y lograr un entendimiento cabal de los valores que determinan la exactitud vertical.

\section{RESULTADOS}

En la Tabla 2 se presenta el resultado que indica y corrobora a que sistema de referencia vertical corresponde los MDE, analizados en este estudio.

A continuación, se describe el Análisis Estadísticos para cada nivelación utilizando el método de exactitud de Diferencias.

Primero se describe, la línea "Valparaíso Santiago", cuyos resultados se indican en Tabla 3 se pudo evaluar valores de diferencias de alturas y error entre los MDE y la nivelación, con un rango de valores para este caso, del orden de $-28 \mathrm{~m}$ y $+10 \mathrm{~m}$ con un promedio de error RMSE de $\pm 11 \mathrm{~m}$

${ }^{20}$ National Standard for Spatial Data Accuracy

Revista Geográfica de Chile Terra Australis, Vol.56 (2020) 110-128, ISSN: 0378-8482 - EISSN: 0719-9562 
de exactitud vertical evaluando los resultados de los 3 MDE.

Segundo la línea "Santiago-Los Andes", cuyos resultados se indican en Tabla 4, por la cual, se pudo evaluar valores de diferencias de alturas y error entre los MDE y la nivelación, con un rango de valores para este caso, del orden de $-23 m$ y $+16 \mathrm{~m}$ con un promedio de error RMSE de $\pm 9 \mathrm{~m}$ de exactitud vertical evaluando los resultados de los 3 MDE.

Tercero la línea "Los Andes-Río Blanco", cuyos resultados se indican en Tabla 5, por la cual, se pudo evaluar valores de diferencias de alturas $y$ error entre los MDE y la nivelación, con un rango de valores para este caso, del orden de -20m y $21 \mathrm{~m}$ con un promedio de error RMSE de $\pm 9 \mathrm{~m}$ de exactitud vertical evaluando los resultados de los 3 MDE.

Cuarto y último que completa la información analizada, es la línea "Río Blanco-Portillo", cuyos resultados se indican en Tabla 6 , por la cual, se pude evaluar valores de diferencias de alturas y error entre los MDE y la nivelación, con un rango de valores para este caso, del orden de $-18 \mathrm{~m}$ y $+15 \mathrm{~m}$ con un promedio de error RMSE de $\pm 6 \mathrm{~m}$ de exactitud vertical evaluando los resultados de los 3 MDE.

\begin{tabular}{|c|c|c|c|}
\hline Sistema de Referencia Vertical & ASTER-GDEM & SRTM & PALSAR \\
\hline EGM-96 & $\mathrm{X}$ & $\mathrm{X}$ & \\
\hline EGM-2008 & & & $\mathrm{X}$ \\
\hline
\end{tabular}

Tabla 2 Comprobación del Sistema de Referencia Vertical: Elaboración Propia

\begin{tabular}{|c|c|c|c|c|c|c|}
\hline \multicolumn{7}{|c|}{ Línea de Nivelación Valparaíso-Santiago } \\
\hline Dem (Fuente abierta) & $\begin{array}{c}\text { Diferencia } \\
\text { Mínima (m) }\end{array}$ & $\begin{array}{c}\text { Diferencia } \\
\text { Máxima }(\mathbf{m})\end{array}$ & $\begin{array}{c}\text { Media } \\
(\mathbf{m})\end{array}$ & $\begin{array}{c}\text { Error } \\
\text { Estándar } \\
\mathbf{d e ~ l a} \\
\text { Media }\end{array}$ & $\begin{array}{c}\text { Desviación } \\
\text { Estándar } \\
(\mathbf{m})\end{array}$ & RMSE (m) \\
\hline ASTER-GDEM & -28 & 10 & -2 & 1.5 & 10 & \pm 10 \\
\hline SRTM & -21 & -1 & -10 & 0.7 & 5 & \pm 11 \\
\hline PALSAR & -21 & 0 & -9 & 0.8 & 6 & \pm 11 \\
\hline
\end{tabular}

Tabla 3 Análisis Estadísticos entre MDE con nivelación geométrica, Método de exactitud de Diferencias. Fuente: Elaboración Propia

\begin{tabular}{|c|c|c|c|c|c|c|}
\hline \multicolumn{7}{|c|}{ Línea de Nivelación Santiago -Los Andes } \\
\hline Dem (Fuente abierta) & $\begin{array}{c}\text { Diferencia } \\
\text { Mínima (m) }\end{array}$ & $\begin{array}{c}\text { Diferencia } \\
\text { Máxima (m) }\end{array}$ & $\begin{array}{c}\text { Media } \\
(\mathbf{m})\end{array}$ & $\begin{array}{c}\text { Error } \\
\text { Estándar } \\
\text { de la } \\
\text { Media }\end{array}$ & $\begin{array}{c}\text { Desviación } \\
\text { Estándar } \\
(\mathbf{m})\end{array}$ & RMSE (m) \\
\hline ASTER-GDEM & -23 & 16 & -3 & 1.4 & 8 & \pm 8 \\
\hline SRTM & -18 & 7 & -8 & 0.7 & 4 & \pm 9 \\
\hline PALSAR & -19 & 9 & -8 & 0.7 & 4 & \pm 9 \\
\hline
\end{tabular}

Tabla 4 Análisis Estadísticos entre MDE con nivelación geométrica, Método de exactitud de Diferencias. Fuente: Elaboración Propia 


\begin{tabular}{|c|c|c|c|c|c|c|}
\hline \multicolumn{7}{|c|}{ Línea de Nivelación Los Andes - Río Blanco } \\
\hline Dem (Fuente abierta) & $\begin{array}{c}\text { Diferencia } \\
\text { Mínima (m) }\end{array}$ & $\begin{array}{c}\text { Diferencia } \\
\text { Máxima }(\mathbf{m})\end{array}$ & $\begin{array}{c}\text { Media } \\
(\mathbf{m})\end{array}$ & $\begin{array}{c}\text { Error } \\
\text { Estándar } \\
\text { de la } \\
\text { Media }\end{array}$ & $\begin{array}{c}\text { Desviación } \\
\text { Estándar } \\
(\mathbf{m})\end{array}$ & RMSE (m) \\
\hline ASTER-GDEM & 13 & -12 & -3 & 1.2 & 6 & \pm 6 \\
\hline SRTM & 14 & -21 & -9 & 1.3 & 7 & \pm 11 \\
\hline PALSAR & -20 & -3 & -9 & 0.8 & 4 & \pm 10 \\
\hline
\end{tabular}

Tabla 5 Análisis Estadísticos entre MDE con nivelación geométrica, Método de exactitud de Diferencias. Fuente: Elaboración Propia

\begin{tabular}{|c|c|c|c|c|c|c|}
\hline \multicolumn{7}{|c|}{ Línea de Nivelación Río Blanco - Portillo } \\
\hline Dem (Fuente abierta) & $\begin{array}{c}\text { Diferencia } \\
\text { Mínima (m) }\end{array}$ & $\begin{array}{c}\text { Diferencia } \\
\text { Máxima (m) }\end{array}$ & Media (m) & $\begin{array}{c}\text { Error } \\
\text { Estándar de } \\
\text { la Media }\end{array}$ & $\begin{array}{c}\text { Desviación } \\
\text { Estándar } \\
(\mathbf{m})\end{array}$ & $\begin{array}{c}\text { RMSE } \\
(\mathbf{m})\end{array}$ \\
\hline ASTER-GDEM & -14 & 10 & 0 & 1.2 & 6 & \pm 6 \\
\hline SRTM & -12 & 7 & -4 & 1.2 & 6 & \pm 7 \\
\hline PALSAR & -18 & 15 & -3 & 1.3 & 6 & \pm 7 \\
\hline
\end{tabular}

Tabla 6 Análisis Estadísticos entre MDE con nivelación geométrica, Método de exactitud de Diferencias. Fuente: Elaboración Propia

Los análisis comprendieron 48 Mediciones para "Valparaíso-Santiago", 35 para "Santiago-Los Andes", 25 en "Los Andes - Río Blanco" y 24 muestras en "Río Blanco - Portillo", el cual contempla un global de 132 muestras distribuidas espacialmente en longitud aproximada $116 \mathrm{~km}$.

En resumen, los resultados en forma global y de acuerdo con los valores desarrollados a partir del método regresión polinómica, teniendo en cuenta un nivel de confianza de $95 \%$ y cuyo resultado de $\left(r^{2}\right)$ coeficiente de determinación ajustado, fue del orden de 0.9999 para cada iteración. De acuerdo con ello, se presentan los resultados en la Tabla 7 .

Los resultados obtenidos indican que analizando su valor promedio con respecto a los MDE es de $\pm 5 \mathrm{~m}$, comparado con el método de exactitud de diferencias es de $\pm 9 \mathrm{~m}$, esto demuestra un ajuste en la exactitud vertical, de un valor de $\pm 4 \mathrm{~m}$ aproximadamente.

\begin{tabular}{|c|c|c|c|}
\hline $\begin{array}{c}\text { Línea de } \\
\text { nivelación }\end{array}$ & $\begin{array}{c}\text { ASTER- } \\
\text { GDEM } \\
(\mathbf{m})\end{array}$ & $\begin{array}{c}\text { SRTM } \\
(\mathbf{m})\end{array}$ & $\begin{array}{c}\text { PALSAR } \\
(\mathbf{m})\end{array}$ \\
\hline $\begin{array}{c}\text { Valparaíso- } \\
\text { Santiago }\end{array}$ & \pm 9 & \pm 4 & \pm 5 \\
\hline $\begin{array}{c}\text { Santiago-Los } \\
\text { Andes }\end{array}$ & \pm 7 & \pm 4 & \pm 4 \\
\hline $\begin{array}{c}\text { Los Andes-Rio } \\
\text { Blanco }\end{array}$ & \pm 6 & \pm 6 & \pm 4 \\
\hline $\begin{array}{c}\text { Rio Blanco- } \\
\text { Portillo }\end{array}$ & \pm 6 & \pm 5 & \pm 6 \\
\hline
\end{tabular}

Tabla 7 Análisis Estadísticos entre MDE con nivelación geométrica, Método de Regresión Polinómica. Fuente: Elaboración Propia

De acuerdo de caso a caso se puede indicar que los valores del método de exactitud de diferencias muestra valores entre $\pm 6 \mathrm{~m}$ y $\pm 11 \mathrm{~m}$ de error en exactitud vertical RMSE en cambio el método de regresión polinómica establece

Revista Geográfica de Chile Terra Australis, Vol.56 (2020) 110-128, ISSN: 0378-8482 - EISSN: 0719-9562 
valores entre $\pm 4 \mathrm{~m}$ y $\pm 9 \mathrm{~m}$, en consecuencia, este análisis, alcanzo una corrección altitudinal en un $41 \%$, para la nivelación Valparaíso Santiago, un $45 \%$ para el caso de Santiago-Los Andes, además en Los Andes - Río Blanco de un $42 \%$ y en la línea final Río Blanco Portillo de un $16 \%$.

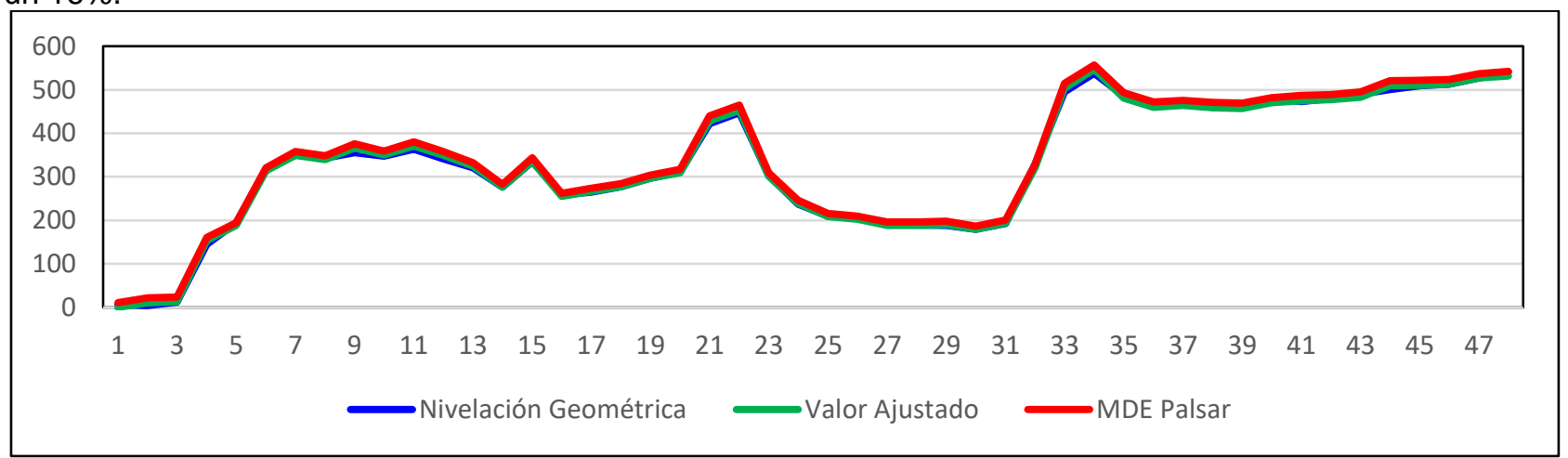

Figura 5 Línea de Nivelación Valparaíso-Santiago. Este gráfico representa la diferencia altitudinal para el modelo PALSAR, capturado por sistema de radar y se considera en color verde los datos ajustados utilizando la regresión polinómica, a continuación, se despliega los parámetros de ajuste utilizado. Fuente: Elaboración Propia.

$$
f(y)=-12.2902270947+1.0436574457 . x+0.0000323550 . x^{2}+-0.0000003588 x^{3}+0.0000000005 x^{4}
$$

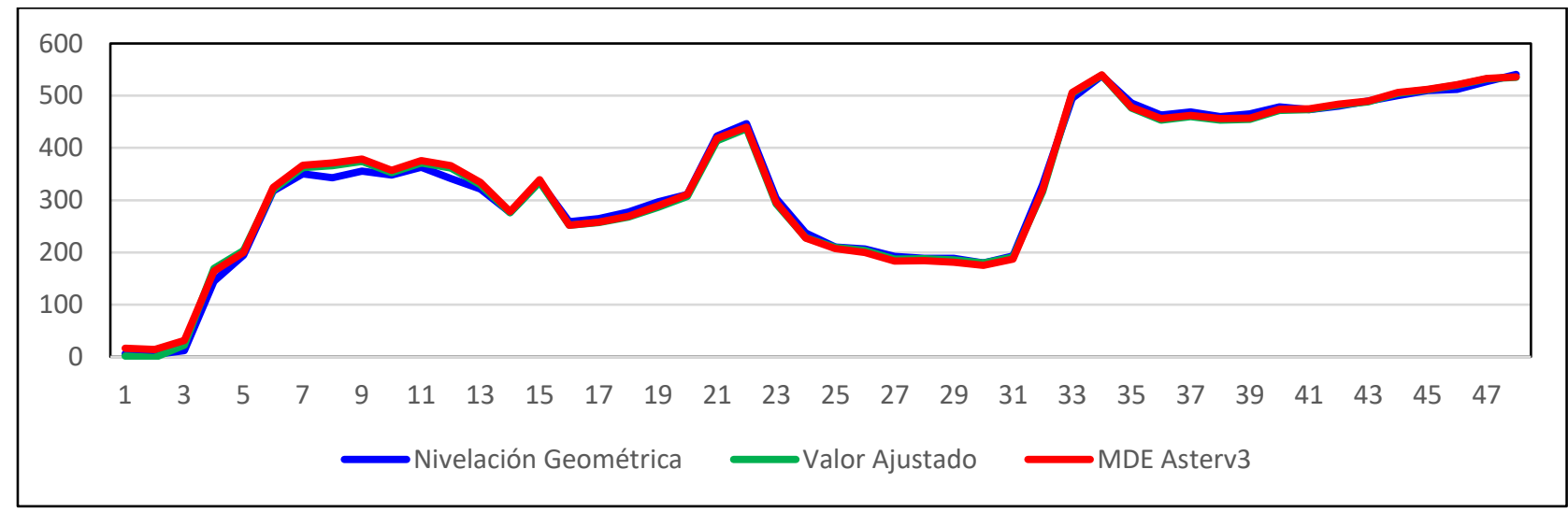

Figura 6 Línea de Nivelación Valparaíso-Santiago. Este gráfico representa la diferencia altitudinal para el modelo ASTER-GDEM, capturado por Sistema óptico y se considera en color verde los datos ajustados utilizando la regresión polinómica, a continuación, se despliega los parámetros de ajuste utilizado. Fuente: Elaboración Propia

$$
f(y)=-21.715084138+1.494806229 . x+-0.002942810 . x^{2}+0.000006390 x^{3}+-0.000000005 . x^{4}
$$

Revista Geográfica de Chile Terra Australis, Vol.56 (2020) 110-128, ISSN: 0378-8482 - EISSN: 0719-9562 


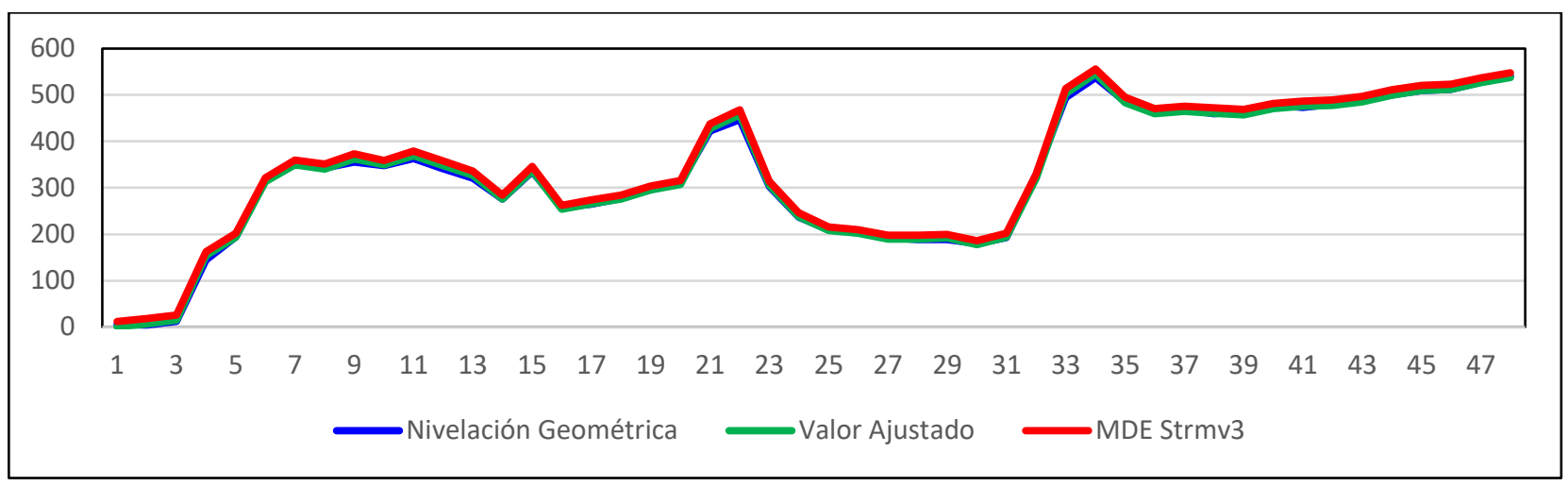

Figura 7 Línea de Nivelación Valparaíso-Santiago. Este gráfico representa la diferencia altitudinal para el modelo SRTM, capturado por sistema de radar y se considera en color verde los datos ajustados utilizando la regresión polinómica, a continuación, se despliega los parámetros de ajuste utilizado. Fuente: Elaboración Propia

$$
f(y)=0.9990628335+-11.4492498103 . x+0.0001545920 . x^{2}+-0.0000007840 . x^{3}+0.0000000009 . x^{4}
$$

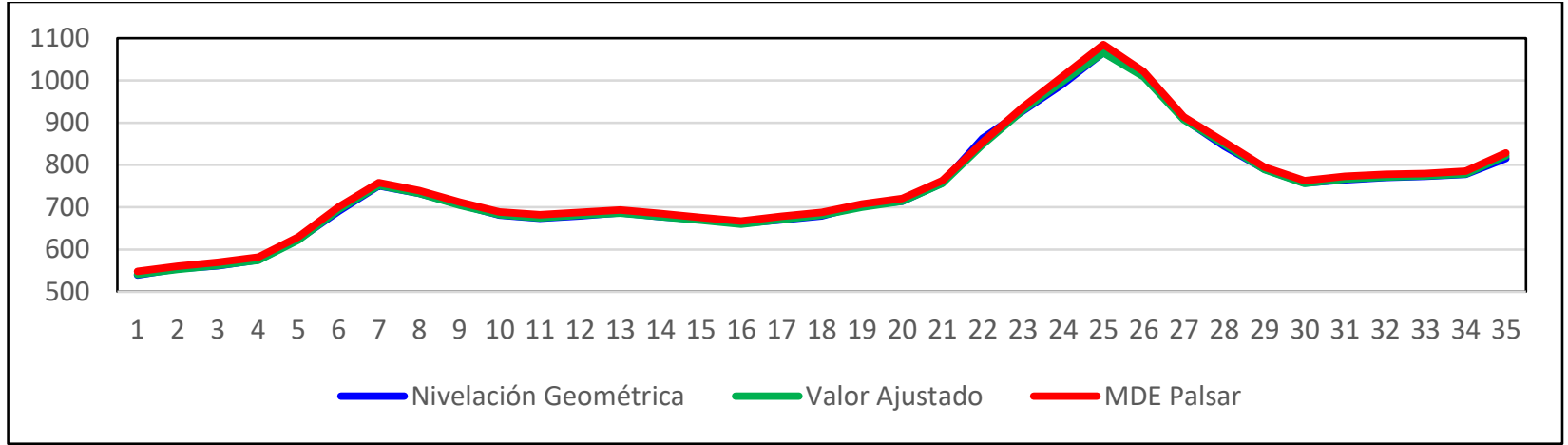

Figura 8 Línea de Nivelación Santiago-Los Andes. Este gráfico representa la diferencia altitudinal para el modelo PALSAR, capturado por sistema de radar y se considera en color verde los datos ajustados utilizando la regresión polinómica, a continuación, se despliega los parámetros de ajuste utilizado. Fuente: Elaboración Propia

$f(y)=31.9941708873+0.9002393200 \cdot x+-0.0000706191 \cdot x^{2}+-0.0000706191 \cdot x^{3}+-0.0000000002 \cdot x^{4}$

Revista Geográfica de Chile Terra Australis, Vol.56 (2020) 110-128, ISSN: 0378-8482 - EISSN: 0719-9562 


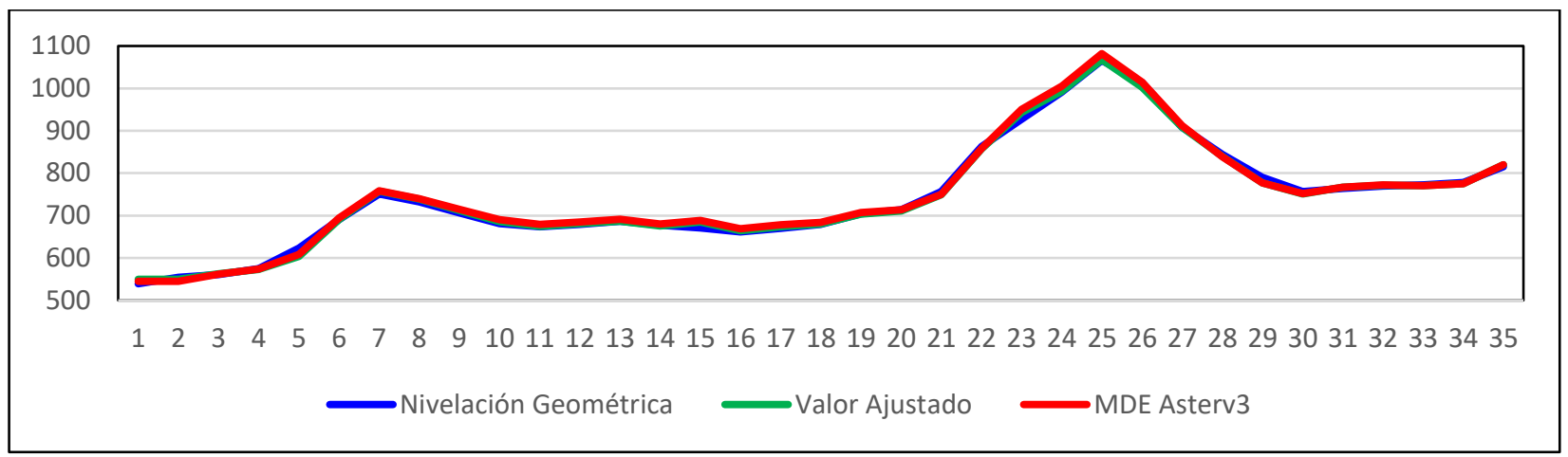

Figura 9 Línea de Nivelación Santiago-Los Andes. Este gráfico representa la diferencia altitudinal para el modelo ASTER-GDEM, capturado por Sistema óptico y se considera en color verde los datos ajustados utilizando la regresión polinómica, a continuación, se despliega los parámetros de ajuste utilizado. Fuente: Elaboración Propia

$$
f(y)=2089.289881164+-9.618638358 \cdot x+0.019801566 \cdot x^{2}+-0.000016067 \cdot x^{3}+0.000000005 \cdot x^{4}
$$

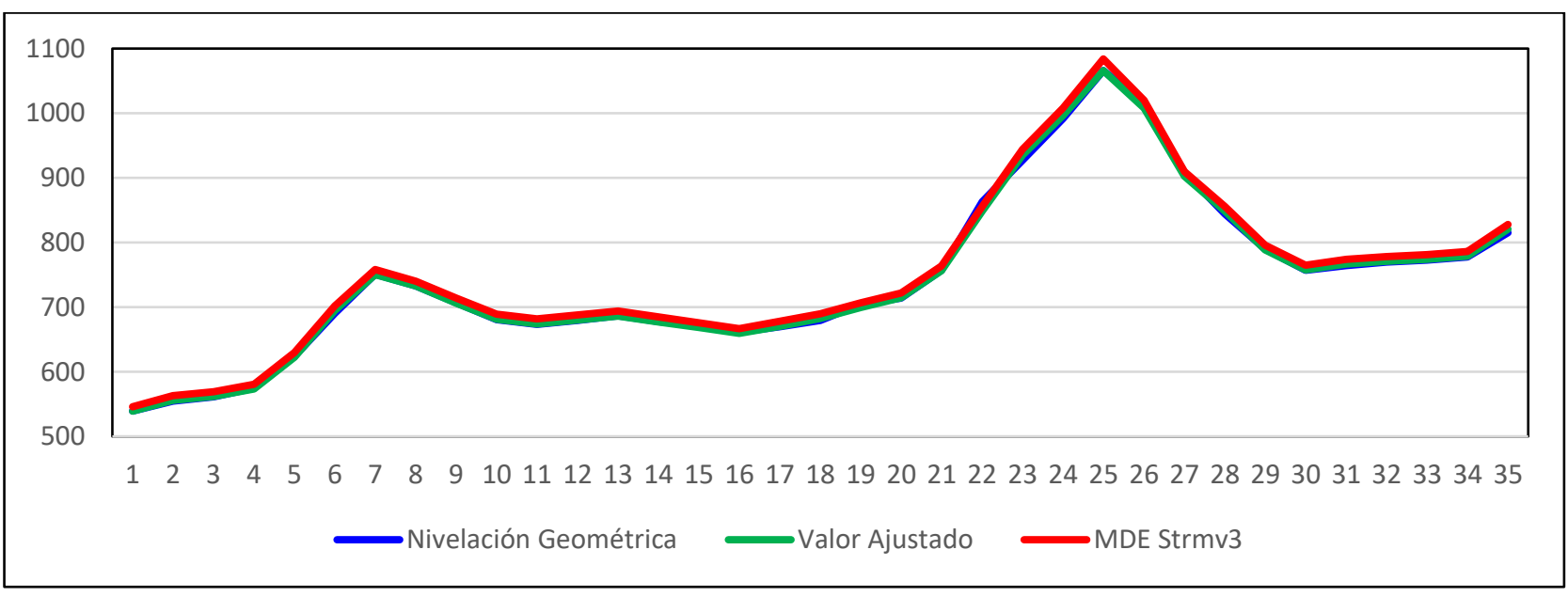

Figura 10 Línea de Nivelación Santiago-Los Andes. Este gráfico representa la diferencia altitudinal para el modelo SRTM, capturado por sistema de radar y se considera en color verde los datos ajustados utilizando la regresión polinómica, a continuación, se despliega los parámetros de ajuste utilizado. Fuente: Elaboración Propia

$$
f(y)=0.3616058312+1.0789176718 \cdot x+-0.0004174920 . x^{2}+0.0000006098 \cdot x^{3}+-0.0000000003 \cdot x^{4}
$$

Revista Geográfica de Chile Terra Australis, Vol.56 (2020) 110-128, ISSN: 0378-8482 - EISSN: 0719-9562 


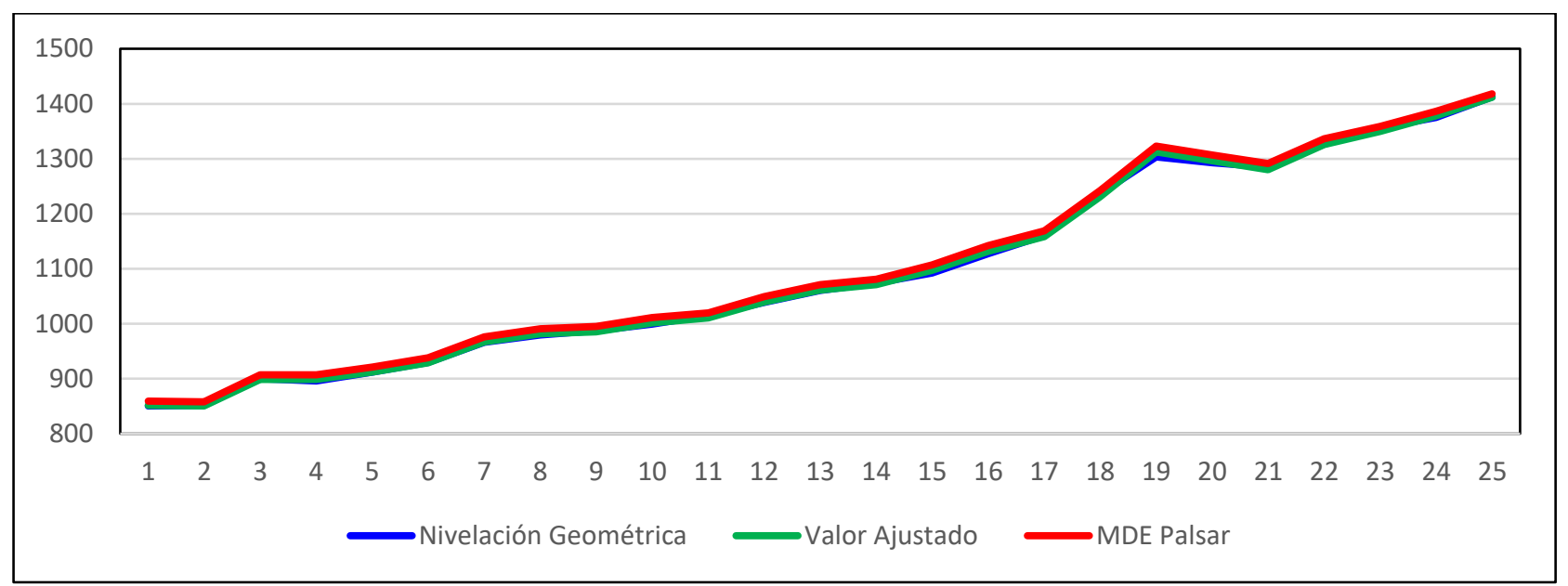

Figura 11 Línea de Nivelación Los Andes- Río Blanco. Este gráfico representa la diferencia altitudinal para el modelo PALSAR, capturado por sistema de radar y se considera en color verde los datos ajustados utilizando la regresión polinómica, a continuación, se despliega los parámetros de ajuste utilizado. Fuente: Elaboración Propia

$$
f(y)=854.279167383+-2.190557361 \cdot x+0.004422711 \cdot x^{2}+-0.000002723 \cdot x^{3}+0.000000001 \cdot x^{4}
$$

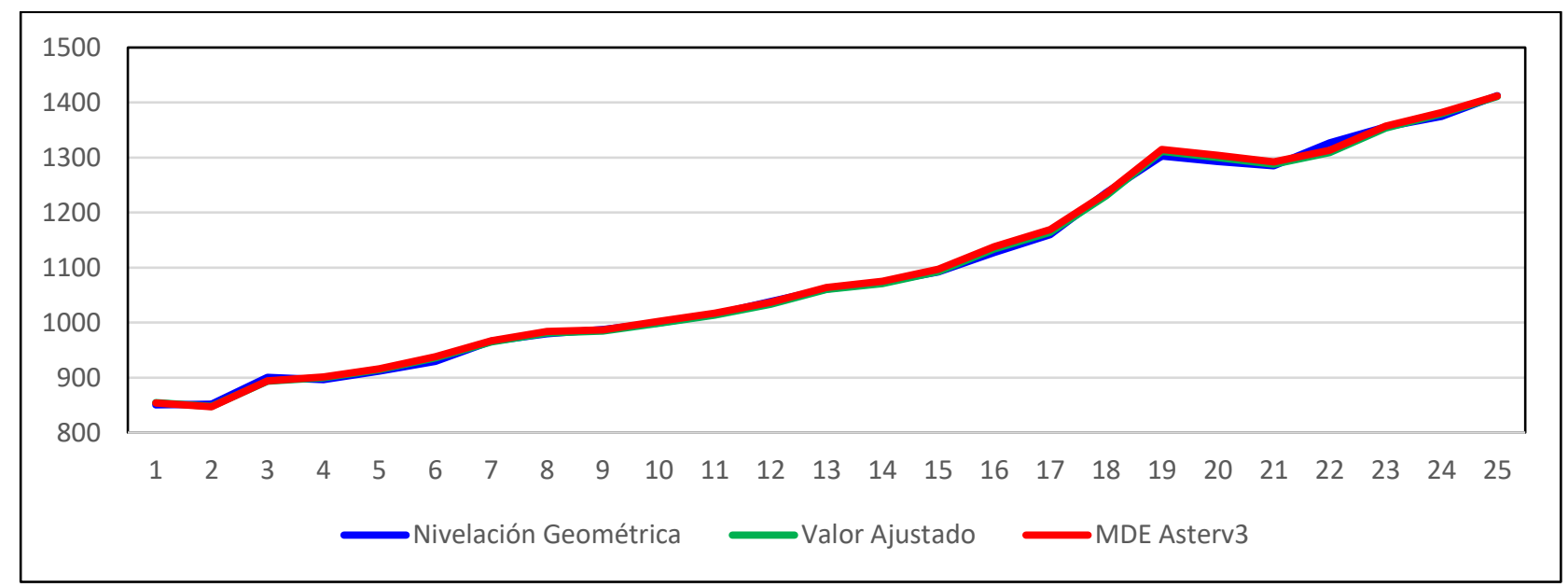

Figura 12 Línea de Nivelación Los Andes- Río Blanco. Este gráfico representa la diferencia altitudinal para el modelo ASTER-GDEM, capturado por Sistema óptico y se considera en color verde los datos ajustados utilizando la regresión polinómica, a continuación, se despliega los parámetros de ajuste utilizado. Fuente: Elaboración Propia

$$
f(y)=511.7257036247+-0.8271114906 . x+0.0024713720 . x^{2}+-0.0000015059 . x^{3}+0.0000000003 \cdot x^{4}
$$

Revista Geográfica de Chile Terra Australis, Vol.56 (2020) 110-128, ISSN: 0378-8482 - EISSN: 0719-9562 


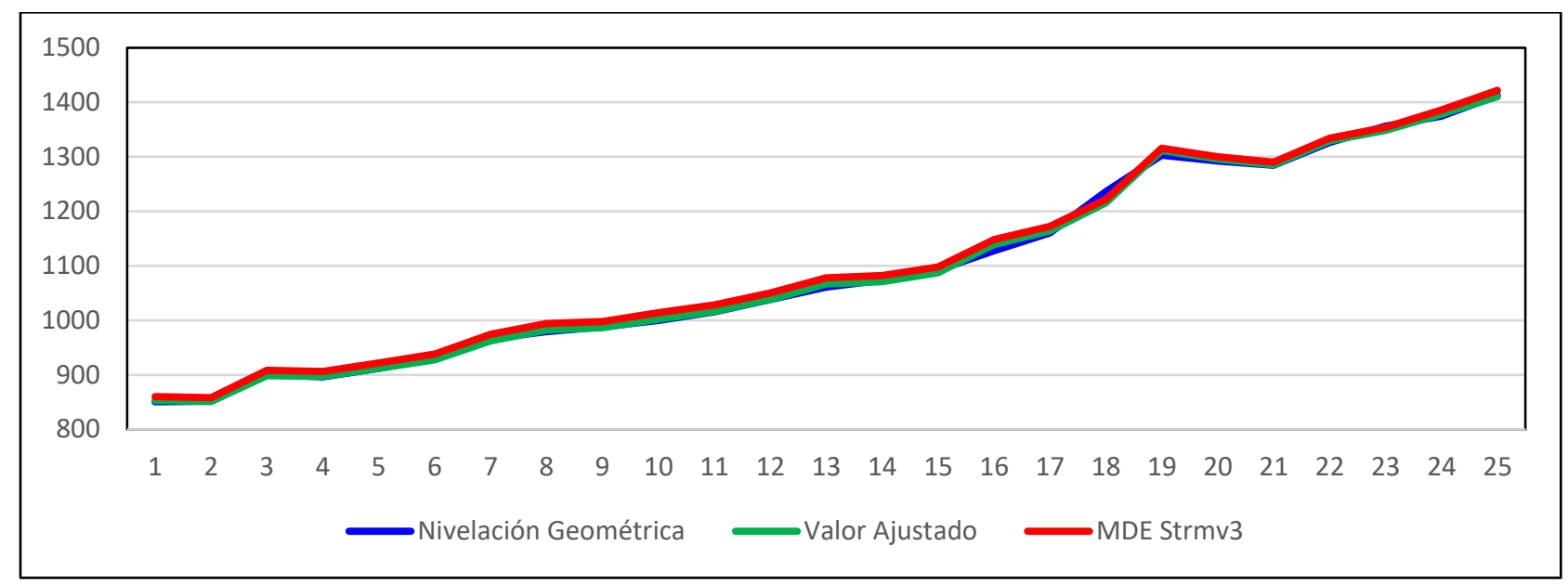

Figura 13 Línea de Nivelación Los Andes- Río Blanco. Este gráfico representa la diferencia altitudinal para el modelo SRTM, capturado por sistema de radar y se considera en color verde los datos ajustados utilizando la regresión polinómica, a continuación, se despliega los parámetros de ajuste utilizado. Fuente: Elaboración Propia

$$
f(y)=-873.246964776+4.850241345 \cdot x+-0.006154971 \cdot x^{2}+0.000004214 \cdot x^{3}+-0.000000001 \cdot x^{4}
$$

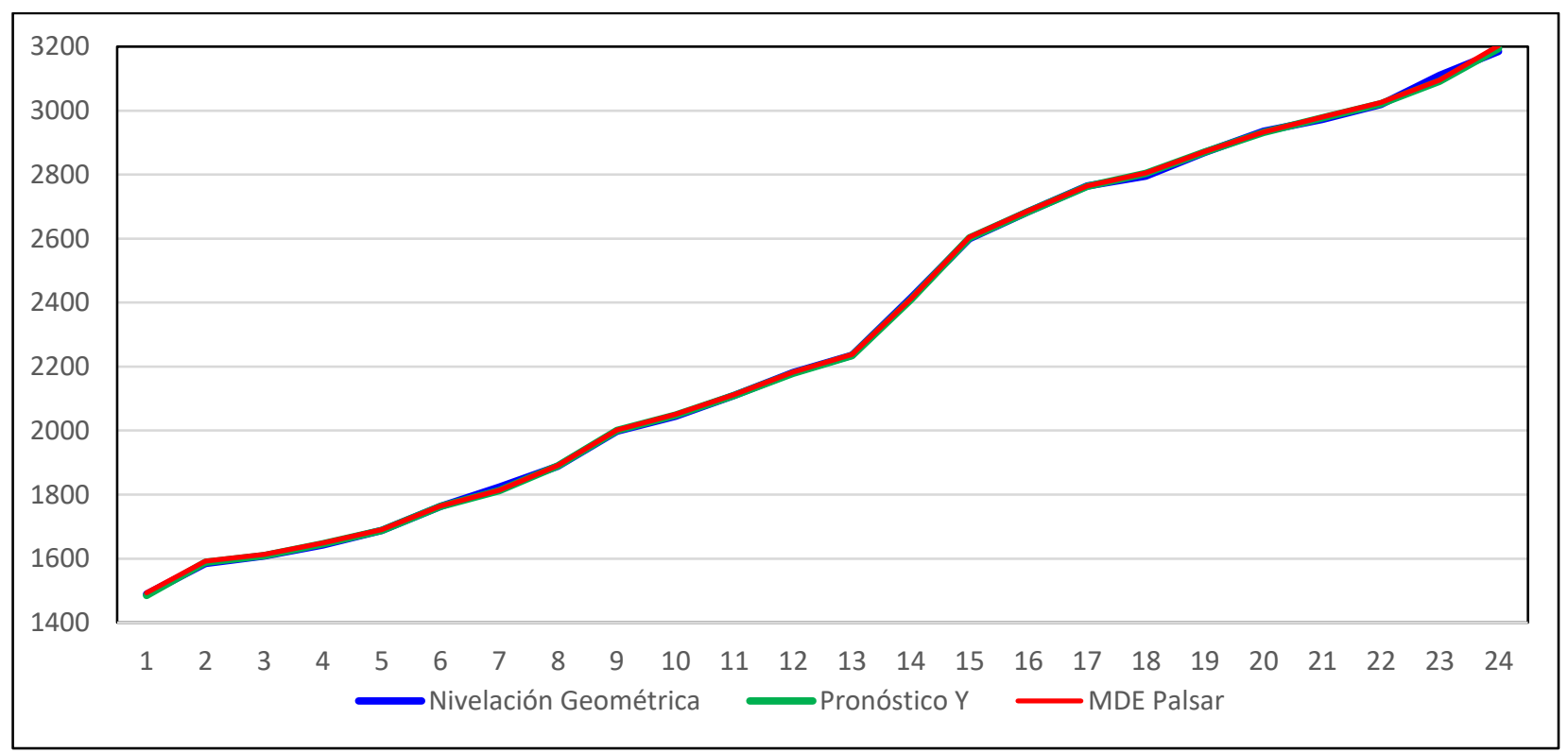

Figura 14 Línea de Nivelación Río Blanco - Portillo. Este gráfico representa la diferencia altitudinal para el modelo PALSAR, capturado por sistema de radar y se considera en color verde los datos ajustados utilizando la regresión polinómica, a continuación, se despliega los parámetros de ajuste utilizado. Fuente: Elaboración Propia

Revista Geográfica de Chile Terra Australis, Vol.56 (2020) 110-128, ISSN: 0378-8482 - EISSN: 0719-9562 


$$
\begin{gathered}
f(y)=-985.76573492+2.7627158307 \cdot x+-0.00116297243 \cdot x^{2}+0.00000033506 \cdot x^{3} \\
+-0.00000000004 \cdot x^{4}
\end{gathered}
$$

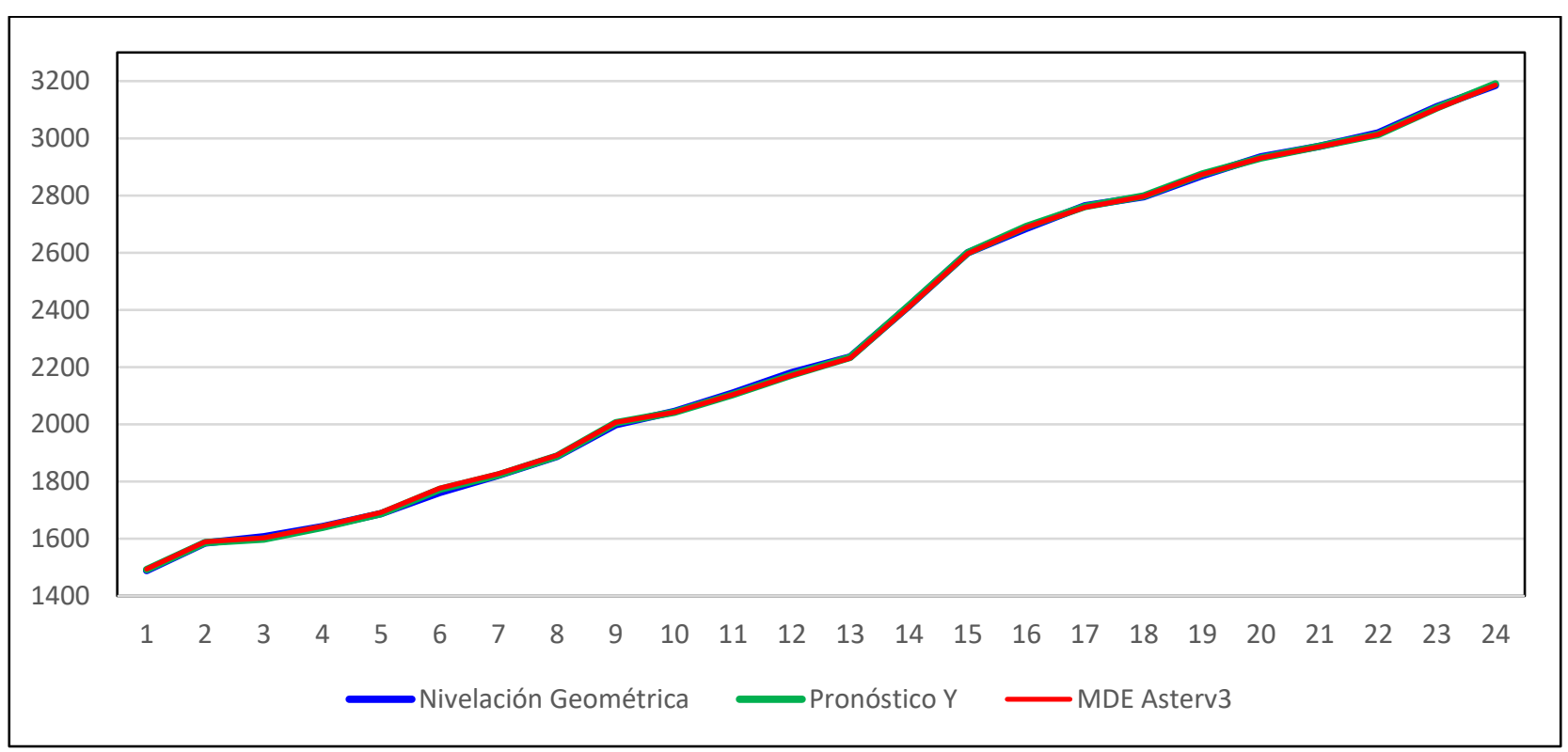

Figura 15 Línea de Nivelación Río Blanco - Portillo. Este gráfico representa la diferencia altitudinal para el modelo ASTER-GDEM, capturado por Sistema óptico y se considera en color verde los datos ajustados utilizando la regresión polinómica, a continuación, se despliega los parámetros de ajuste utilizado. Fuente: Elaboración propia.

$$
\begin{gathered}
f(y)=869.912536446+-0.60726541919 . x+0.00107777195 \cdot x^{2}+-0.00000031205 \cdot x^{3} \\
+0.00000000003 \cdot x^{4}
\end{gathered}
$$

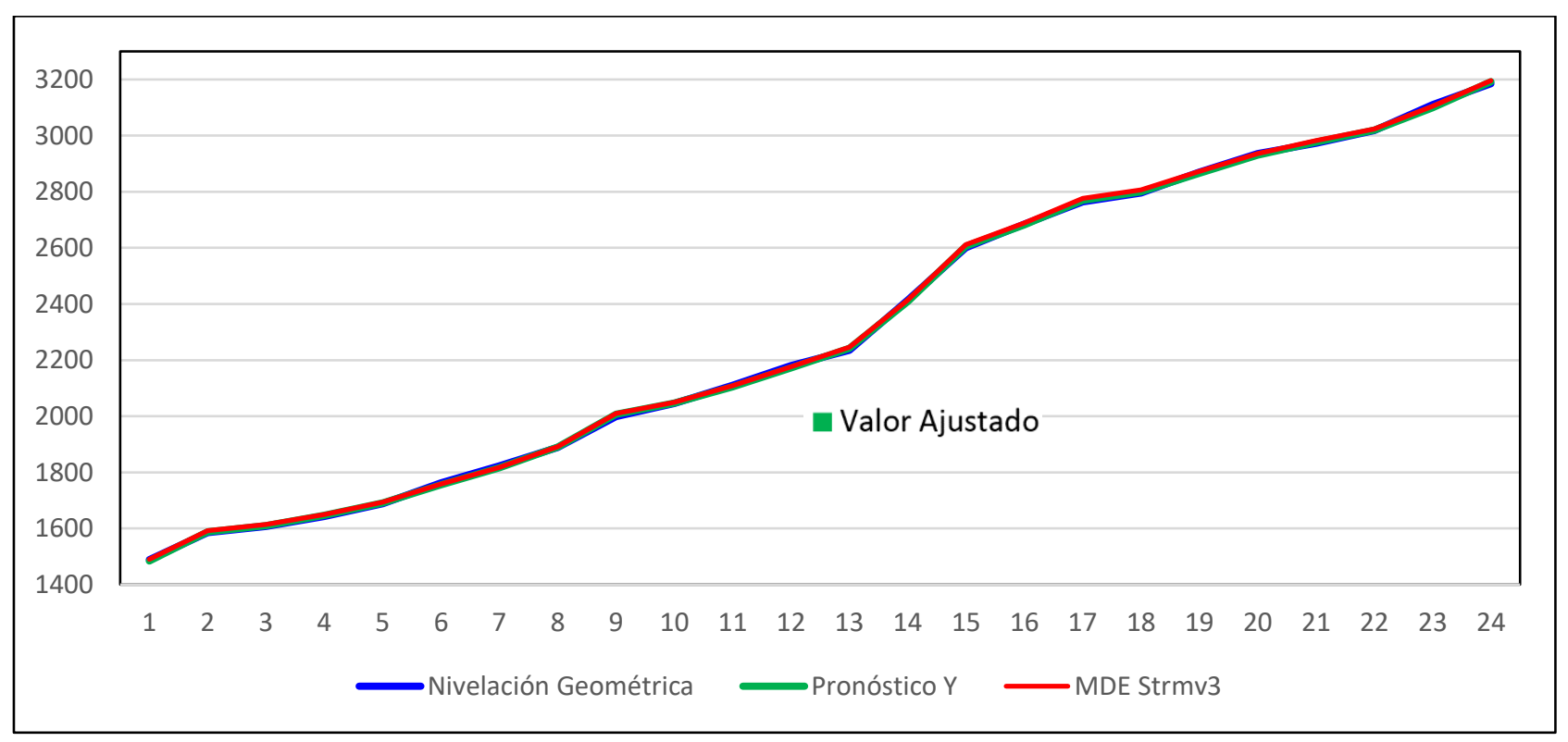

Revista Geográfica de Chile Terra Australis, Vol.56 (2020) 110-128, ISSN: 0378-8482 - EISSN: 0719-9562 
Figura 16 Línea de Nivelación Río Blanco - Portillo. Este gráfico representa la diferencia altitudinal para el modelo SRTM, capturado por sistema de radar y se considera en color verde los datos ajustados utilizando la regresión polinómica, a continuación, se despliega los parámetros de ajuste utilizado. Fuente: Elaboración Propia

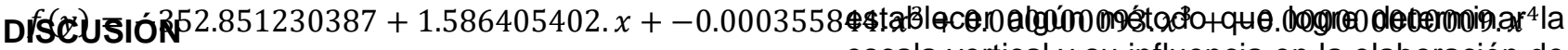 escala vertical y su influencia en la elaboración de \\ De acuerdo con Martínez et al., (2014), indica que \\ las curvas de nivel.} el mayor error se encuentra en terrenos de alta pendiente mientras que en zonas de pendiente baja sucede lo contrario, ahora bien, en este estudio los datos en la zona de terreno más alta "Río blanco - Portillo", este, arrojo totalmente lo inverso entrego la exactitud más baja. Esto influencia en analizar e involucrar las pendientes como una variable de análisis para alcanzar mejores resultados en los diferentes estudios realizados en orden de la exactitud vertical.

En Chile, existen pocos artículos científicos publicados, de acuerdo con la temática expuesta en este trabajo. A partir de este desconocimiento se generan oportunidades de mejora e implementación de metodologías que aporten en difundir nuevos productos. Para que estos, sean utilizados en fortalecer estudios que se desarrollan en el contexto país, ya que es información gratuita, de fácil acceso y de calidad adecuada a la escala específica que se quiera trabajar.

Es importante señalar que en relación con el manejo del método de regresión polinómica que evalúa los factores locales de ajuste, cabría preguntarse, como lograr ajustar más territorio. Sobre este asunto, se recomienda la posible sectorización, utilizando ciertos criterios, asimismo, de disponer de una malla de puntos distribuidas homogéneamente para cubrir mayor territorio. Lo anterior expuesto busca discretizar áreas mayores de topografía local para que mantenga cierta heterogeneidad y aporte finalmente en una mejor exactitud de la información resultante.

Se puede indicar que, en la presente investigación, se evaluaron fuentes de datos disímiles y con resoluciones espacial que oscilan entre $12.5 \mathrm{~m}$ a $28 \mathrm{~m}$. De acuerdo con los datos enunciados se abre una incertidumbre para determinar la escala real horizontal y vertical. Este tema es relevante y se recomienda hacer estudios, esta visión, busca
Con este trabajo no se pretende agotar la temática de análisis, en consecuencia, este busca ampliar el conocimiento en post de la creación de nuevos artículos científicos que experimenten, por ejemplo: otras líneas de nivelación geométrica evaluadas con otros modelos digitales (LIDAR, TANDEM). Asimismo, la utilización y verificación de modelos digitales de elevación, elaborados a partir de cartografía regular IGM.

\section{CONCLUSIONES}

Lo expuesto a lo largo de este trabajo permite indicar que la determinación de parámetros de ajuste locales fue llevada a cabo, mediante la aplicación del método de la ecuación de observación deducida a partir de una regresión polinómica. En conclusión, los resultados indican que el empleo de dicha ecuación aporta en el ajuste de la exactitud vertical comparado con un valor de mayor precisión. Cabe indicar que dicha formula de ajuste se utiliza en procesos de exactitud vertical y otorga valores de ajustes locales, es decir, al área que comprende la muestra analizada.

Según los resultados encontrados, se precisa que a mayor altitud se refleja un descenso en el error de exactitud vertical con el método de exactitud de diferencias ya que el análisis demuestra un descenso en los promedios del RMSE $\pm 4 \mathrm{~m}$, evaluando, la línea "Valparaíso-Santiago" iniciando a nivel del mar con la de cordillera "RíoBlanco-Portillo". Sin embargo, en el sector central determinado por las líneas "Santiago-Los Andes" y "Los Andes Río Blanco" no existe diferencia, es decir presente un mismo comportamiento.

En el marco de este artículo académico, se puede concluir que los modelos que contiene menos errores sistemáticos y tiende a correlacionarse son SRTM y ALOS PALSAR, ellos están elaborados por Interferometría Radar, a diferencia del sensor

Revista Geográfica de Chile Terra Australis, Vol.56 (2020) 110-128, ISSN: 0378-8482 - EISSN: 0719-9562 
óptico el Aster-Gdem que presenta más errores anómalos.

Los temas tratados en esta publicación son la exactitud y la precisión vertical sobre los MDE de fuente abierta, a este respecto, se evidenció que los máximos errores sistemáticos para la zona central de Chile están referidos en sectores determinados por el efecto del relieve tales como: valles, cimas de montañas y elementos antrópicos, por los cuales, se vislumbra que es debido a los sesgos por captura y procesamiento del sensor óptico y radar. Visto de esta forma, estos errores son influenciados por efecto de sombra, distorsión e inversión por relieve, al momento de captura satelital de los datos. Dentro de ese contexto, quiero indicar que los modelos globales, presentan errores propios ya que están automatizados y sin mucho control, una alternativa seria tener por ejemplo un modelo de fuente radar, pero con la interferometría ejecutada con control en tierra, lo más probable es que tanto el comportamiento como los errores propios de su metodología permitiría mejorar la estimación en la precisión de la información relevada.

Los resultados indican que existen desviaciones altimétricas en las 4 nivelaciones con respecto a la superficie real, determinada por la nivelación geométrica. Eso define que los tres modelos tienen errores, de acuerdo con ello, se recomienda hacer estudios más acabado para caracterizar el origen de los errores con una mayor determinación.

\section{REFERENCIAS}

Alganci, U., Besol, B., \& Sertel, E. (2018). Accuracy Assessment of Different Digital Surface Models. ISPRS International Journal of Geo-Information.

Bolla, A. M. (2009). Geodesia y Cartografía. Catalunya: Universitat Oberta de Catalunya.

Burgos, V. H. (10 de 6 de 2012). Evaluación de ASTER GDEM y SRTM-C/X para modelación hidráulica de la rotura de presa El Carrizal, Mendoza. Obtenido de https://www.researchgate.net/publication/2748934 $62 \% 0$ AEvaluación
C. Brunini, C., Sanchez, L., Martínez-Díaz, W., Luz, R., \& Mackern, M. (01 de 10 de 2011). Sistema de Referencia Geocéntrico para Las Américas. Obtenido de http://www.sirgas.org/fileadmin/docs/Boletines/Bol etin_SIRGAS_No_16.pdf

Doyle, F. (1978). Digital terrain models: an overview. Photo- grammetric Engineering and Remote Sensing, 1481-1485.

Elkhrachy, I. (14 de 10 de 2018). Vertical accuracy assessment for SRTM and ASTER Digital Elevation. Ain Shams Engineering Journal, 9(4), 1807-1817.

doi:https://doi.org/10.1016/j.asej.2017.01.007.

Esri. (22 de 11 de 2019). Arcgis Resouces. Obtenido https://resources.arcgis.com/es/help/gettingstarted/articles/026n00000014000000.htm

ESRI. (05 de 11 de 2019). Arcmap. Obtenido de https://desktop.arcgis.com/es/arcmap/latest/tools/s patial-analyst-toolbox/extract-values-to-points.htm

Felicísimo, A. M. (1 de Marzo de 1994). Modelos Digitales del Terreno. Oviedo: Universidad de Oviedo. Obtenido

http://www.etsimo.uniovi.es/ feli.

FGDC, F. G. (07 de 03 de 1998). Geospatial Positioning Accuracy Standards, Part 3: National Standard for Spatial Data Accuracy. Obtenido de https://www.fgdc.gov/standards/projects/FGDCstandards-projects/accuracy/part3/chapter3

Françoso, M., Pizarro, I., Almeida, L. d., \& Silva, M. d. (2019). Nivelación Geométrica de Precisión para el Control de Desplazamientos Verticales en la Recuperación y Refuerzo de una Viga de Concreto, Estudio de Caso. Revista Ingeniería de Construcción, 205-214.

Garafulic C., P. (5 de 11 de 2011). Cartografia.cl. Obtenido http://www.cartografia.cl/download/geodesiateorica .pdf

Garafulic, P. (10 de 02 de 2014). Geodesia Teoria y Practica. Obtenido de http://www.cartografia.cl/download/geodesiateorica .pdf

Revista Geográfica de Chile Terra Australis, Vol.56 (2020) 110-128, ISSN: 0378-8482 - EISSN: 0719-9562 
Greenwalt, C. R., \& Shultz, M. E. (1968). Principles of error theory and cartographic applications. St. Louis, Missouri 63118: United States Air Force, Aeronautical Chart and Information Center.

IGM. (5 de 8 de 2019). Departamento de Geodesia. Santiago, Chile.

IGN, I. G. (1 de Enero de 2014). IGN Instituto Geográfico Nacional. Obtenido de Centro Nacional de Informacion Geográfica: https://www.ign.es/web/resources/docs/IGNCnig/G DS-Teoria-Geodesia.pdf

Mancero, H., Toctaguano, D. S., Tacuri, C., \& Kirby, E. P. (2015). Evaluación de Modelos Digitales de Elevación obtenidos por diferentes sensores remotos. 1-6 htpps//doi.org/10.13140/RG.2.1.4777.2325.

Martinez Toro, E. E., \& Bethencourt Fernandez, A. (10 de 01 de 2012). Comparación de la precisión de los modelos geopotenciales globales EGM08 Y EGM96 en la zona del caribe. Obtenido de http://oa.upm.es/22788/1/INVE_MEM_2012_1572 10.pdf

Martínez, C., Reinoso, P., \& Neira, N. (2014). Análisis de exactitud del modelo digital de terreno para Colombia. Revista de Investigacion Universidad de Quindío, 30-38.

Menegbo E. M, D. P. (2015). Vertical accuracy assessment of SRTM3 V2.1 and aster GDEM V2 using. INTERNATIONAL JOURNAL OF GEOMATICS AND GEOSCIENCES.

NG-IA, N.-I. (7 de 8 de 2019). Obtenido de https://earthinfo.nga.mil/GandG/update/index.php? action=home

Rodríguez, F. R., \& Barrena, M. (2006). Indexación de datos SRTM de elevación terrestre. Algoritmos de carga masiva en el árbol Q. XV Jornadas de Ingeniería del Software y Bases de Datos (págs. 57-66). España: CIMNE, Barcelona, 2006.

Saez, C. I. (28 de 6 de 2019). WORKSHOP IDE CHILE 2019. Obtenido de http://www.ide.cl/descargas/Workshop_2019/Pres entaciones/2.SistemaReferenciaChile-TCCristianIturriaga.pptx
Sánchez, L. (10 de 11 de 2002). SIRGAS. Obtenido de

http://www.sirgas.org/fileadmin/docs/Determinacio n_de_alturas_fisicas_en_Colombia.pdf

Sánchez, L. (8 de 6 de 2007). SIRGAS-GTIII: Datum Vertical. Obtenido de http://www.sirgas.org/fileadmin/docs/Boletines/Bol 12/04_Nivel_de_referencia_para_Sirgas_Sanchez .pdf

SIRGAS. (24 de 06 de 2019). Sistema de Referencia Geocéntrico para las Américas (SIRGAS). Obtenido de http://www.sirgas.org/es/sirgas-definition/

SNIT, S. N. (2018). Geodesia en Chile, teoría y aplicación del Sistema de Referencia Geocéntrico para las Américas (SIRGAS). Santiago: Ministerio Bienes Nacionales.

Tierra, A. (10 de 10 de 2009). Evaluación del Egm08 y Egm06 en el Ecuador a Partir de Datos de GPS y Nivelación Geométrica. Obtenido de https://www.researchgate.net/publication/2773321 00\%0AEVALUACIÓN

Zepeda, R. (10 de 11 de 2014). Obtenido de https://www.academia.edu/35816685/GEODESIA_ GEOMÉTRICA

Zepeda, R. (5 de 10 de 2014). Ajuste Geodésico Revisión 3.2. Obtenido de https://es.scribd.com/document/394283219/AJUST E-Rene-Zepeda-Rev3-2-Sep2016 